\title{
Fluoride in cerebrospinal fluid of patients with fluorosis
}

\author{
HU YU-HUAN, WU SI-SHUNG, \\ From the Department of Neurology of the Affiliated Hospital of Shihezi Medical College, Xinjian, Republic of \\ China
}

SUMMARY The CSF fluoride level of individuals drinking water with normal fluoride content and of patients with endemic fluorosis were studied. For the purpose of studying the relationship between the dynamic equilibrium of the CSF fluoride and other body fluids, urine and blood fluoride were examined simultaneously. Fluoride was revealed in every CSF sample of the control group and its mean value was lower than that of the blood. The CSF fluoride concentration of patients with fluorosis was slightly higher than that of the control group, although there was no statistically significant difference. The results suggests that fluoride is a normal component of CSF. In severe cases of fluorosis or breakdown of the blood-brain in some diseases of the central nervous system, the CSF fluoride value might be increased.

We have previously reported the fluoride content of body fluids in individuals drinking water containing higher fluoride concentrations (average level more than $10 \mathrm{ppm}$ ) and of those whose drinking water contained fluoride less than $1 \mathrm{ppm} .{ }^{1}$ The mean value for urinary fluoride concentration of the control group was $0.62 \mathrm{ppm}$. Sex, age and nationalities made no difference. The normal mean concentrations of fluoride in serum and plasma were 0.198 and 0.194 ppm respectively. The mean concentration of urinary fluoride in the patients with fluorosis was $4.5 \mathrm{ppm}$ and that of their serum was $0.25 \mathrm{ppm}$, which are higher than those of the control group. The difference is significant $(p<0.05)$. However, there is overlapping in distribution between the two groups and the result lacks clinically diagnostic significance. These findings are similar to those recently reported elsewhere in China. ${ }^{2-5}$ We have also examined CSF of patients receiving low fluoride water, undergoing lumbar anaesthesia (routine examinations of CSF were normal). Fluoride was detected in every sample and the mean value was $0.17 \mathrm{ppm}$ (table). So far as we know, there has only been one report of CSF fluoride levels. ${ }^{6}$ However, the patients in that report did not suffer from fluorosis and the fluoride concentrations of their drinking water or foods were not mentioned. Moreover, there was no control group in the study. In order to confirm our previous findings and investigate the dynamic relationship between CSF and other body fluids, it is necessary to study further the fluoride levels in CSF, blood and urine in patients with fluorosis.

\section{Patients and method}

Forty one endemic fluorosis patients, aged 14-65, 19 females and 22 males, from districts where fluoride in drinking water was elevated were admitted. All of their clinical fluorosis features were positive. Spot sample of urine fluoride was measured in all patients and serum fluoride in 39 as well as CSF fluoride in 40 patients were determined at the same time. CSF fluoride concentrations in patients undergoing spinal anaesthesia, which had been reported previously with the same method of determination as in this report, were regarded as the control group.

An iron selective electrode technique was used for the determination of the fluoride concentration. This method was used in our previous work and by others in China. ${ }^{3}$

\section{Results}

Table CSF, blood and urine fluoride (ppm)

\begin{tabular}{llllll}
\hline & No & Range & $\bar{X}$ & $S$ \\
\hline \multirow{2}{*}{ CSF of Control Group } & 32 & $0 \cdot 14-0.23$ & 0.17 & 0.03 \\
\multirow{3}{*}{ Fluorosis } & CSF & 40 & $0 \cdot 10-0.36$ & 0.20 & 0.062 \\
& Blood & 39 & $0 \cdot 10-0.38$ & 0.20 & 0.065 \\
& Urine & 41 & $1 \cdot 20-22$ & 5.87 & 3.82 \\
\hline
\end{tabular}




\section{Discussion}

The findings and the data reported previously showed that blood fluoride can be maintained at a steady level even though the urinary fluoride concentration in fluorosis is higher and ranges more widely. The level of CSF fluoride in fluorosis patients was higher than that of the control group, though there is no significant difference $(t=0 \cdot 108, p<0.5)$. It is possible that there is a physiological mechanism to maintain dynamic equilibrium of fluorine metabolism in the body and to keep a steady level of fluoride in the blood and CSF. In 36 patients, CSF and blood fluoride concentrations were examined simultaneously. CSF fluoride concentrations were higher than those of blood in 16 , lower than blood in 18 , and at the same level in two patients. We draw the preliminary conclusion that the fluoride is a normal component of CSF, as it could be detected in every sample whether patient or normal control. In normal individuals, fluoride is in dynamic equilibrium between the blood and CSF, and CSF fluoride concentration is similar to or slightly lower than that in blood. These facts indirectly indicate that the passage of fluorine through the blood-brain barrier is by no means a passive diffusion, but an active transport function which is similar to that of other halogen and ionic substances. The normal CSF/blood ratio is below $1 \cdot 0 .^{7}$ Only in patients with severe fluoride intoxication or the breakdown of the blood-brain barrier, as with some diseases of the central nervous system, would the fluoride content in CSF be increased.

Fluorine is considered to be an essential element. It is stored chiefly in the osseous system. The study of the biological effects of fluoride has centered chiefly on bones, teeth and enzyme systems. There has been little interest in the accumulation and effects of fluoride in the nervous system. Recently, reports have shown normal brain tissues which contain fluoride and its concentration was higher in the hypothalamus and hippocampus than other regions. ${ }^{8}{ }^{9} \mathrm{Kay}$ et al found that anion fluoride could affect calcium conductance by an intracellular action in hippocampal neuron of guinea pigs. ${ }^{10}$ Still et al reported that the incidence of primary degenerative dementia (Alzheimer's disease) in some districts having $1.0 \mathrm{ppm}$ fluoride level in the drinking water was higher than in districts having a higher level of fluoride in the drinking water. They suggested that fluoride might decrease brain aluminium, which is thought to be related to the development of Alzheimer's disease. ${ }^{11}$ However, fluoride metabolism in the central nervous system has not yet been thoroughly and systematically studied.

There have been reports of damage to the nervous system caused by endemic fluorosis and longterm fluoride therapy with dosages of sodium fluoride in some cases as high as $200 \mathrm{mg} /$ day. ${ }^{12-15}$ Interpretation was complicated, however, by changes in the osseous system or its neighbouring structures such as ligament, meninges etc. Whether or not high concentrations of fluoride could directly damage the nervous system (especially the central nervous system) is still not known. We have seen some patients with high body fluoride levels and unclassified nervous lesion of unknown aetiology. After removal from the higher fluoride exposure, fluoride in their body fluid decreased and their symptoms improved. ${ }^{16}$ We thought that fluorine might damage the nervous system directly. Therefore, a determination of CSF fluoride level would be helpful for the diagnosis of fluoride intoxication and would provide some information regarding the physiopathological effect of fluoride on the central nervous system.

\section{References}

$1 \mathrm{Wu}$ Si-Shung. Determination of fluoride content in urine, blood and CSF in normal person and patients with fluorosis (in Chinese). The compilation of First Conference on Neuropsychiatric Diseases in Xinjian 1982:89-91.

2 Xian Medical College. Symposium on Endemic Fluorosis (in Chinese). Xian: Xian Medical College Press, 1985.

3 Du Ming-yuan. Elimination of fluoride in urine as a parameter of fluoride overload in human body, analysis of 2814 cases (in Chinese). Acta Nutrimenta Sinica 1984;6:67-71.

4 Guo Chunsheny. The determination of the normal value of fluoride in Zhemeng of Inner Mongolia (in Chinese). Chinese Journal of Endemiology 1984;3:108-10.

5 Wang Du-sheng. Observation of the serum free fluoride contents in populace in endemic and non-endemic areas with different concentrations of fluoride in drinking water (in Chinese). Acta Academiae Medicine Tianjin 1984;8:1-5.

6 Singer L, Armstrong WD, Lavender DR. Fluoride levels of plasma and cerebrospinal fluid. J Dent Res 1967;46:455.

7 Davison H. The blood-brain barrier. In: Boume QH (ed): The Structure and Function of Nervous Tissue, New York Academic Press, 1972;4:323-35.

8 Steinecke H, Schaper R. Fluoridbestimmung in biologischem material. Krim Forens Wiss 1983;49-50:98-102.

9 Chan AWK, Minski MJ, Lai JCK. An application of neutron activation to small biological samples: simutaneous determination of thirty elements in rat brain regions. J Neurosci Methods 1983;7:317-29.

10 Kay AR, Miles R, Wong RKS. Intracellular fluoride alters the kinetic properties of calcium currents facilitating the investigation of synaptic events in hippocampal neurons. J Neurosci 1986;6:2915-20.

11 Still CN, Kelley P. On the incidence of primary degenerative dementia vs. water fluoride content in South Carolina. Neurotoxicology 1980;1:125-31. 
12 Singh A, Jolly SS, Bansal BC. Skeletal fluorosis and its neurological complications. Lancet 1961;i:197-200.

13 Riggs BL, Hodgson DL, Kelly PJ, Johnson KA, Taves D. Treatment of primary osteoporosis with fluoride and calcium, clinical tolerance and fracture occurrence. JAMA 1980;243:446-9.

14 Shung-Guan Cu-min. The nonskelectal lesion of endemic fluorosis (in Chinese). Chinese J Inter Med
1982;21:217-9.

15 Ding Lou-Ian. The nervous systemic complication of chronic fluorosis (in Chinese). Chinese Journal of Endemiology 1983;2:97-8.

$16 \mathrm{Hu}$ Yu-huan. Direct damage on nervous system by fluorosis, (in Chinese). The Compilation of The First Conference on Neuropsychiatric Diseases in Xinjian 1982:86-8.

\section{Little's disease}

Every student is taught about Little's spastic diplegia, but how many know that William John Little (1810-94) was himself afflicted with a congenital equinus deformity of his left foot? He was founder of the Royal Orthopaedic Hospital and Senior Physician to the London Hospital. His several disquisitions ${ }^{1-3}$ are quite brilliant and repay study in full.

"I have witnessed so many cases of deformity, mental and physical, traceable to causes operative at birth, that I consider the subject worthy of notice ${ }^{3}$ of the Obstetrical Society." He mentions 200 cases encountered in 20 years of orthopaedic practice. He stressed the "larger proportion of dead, stillborn, apoplectic, or asphyxiated at birth have been rendered so by interruption of the proper placental relation of the foetus to the mother, and non-substitution of pulmonary respiration, than from direct mechanical injury to the brain and spinal cord." The consequences were "internal congestions, capillary extravasations, serous effusions which correspond with. . . asphyxia, suspended animation, apoplexy, torpidity, tetanic spasms, convulsions of newborn children, and the spastic rigidity, paralysis, and idiocy subsequently witnessed."

"The flexors and abductors of the thighs, flexors of the knees, and the gastrocnemii preponderate. . thighs cannot be completely abducted or extended, the knees cannot be straightened, nor the heels applied to the ground. The elbows are semiflexed, wrists partially flexed and pronated and fingers incapable of perfect voluntary direction. . . upper extremities sometimes appear unaffected. . . Muscles of speech are commonly involved,. . . articulation is slow and difficult. . . in the majority of cases the intellect suffers - from the slightest impairment up to entire imbecility."

Little relates presentations with convulsions, opisthotonus or laryngismus in the early days and the delay in recognising paralysis until the child starts to walk in milder cases; he describes the deformities of posture, trunk and the impediment in walking. He had sparse necropsy evidence "Case LX showing cicatrised apoplexies on surface and interior surfaces of the brain. . . effusion of blood in both ventricles of brain-a true apoplexy (case XLI-a precipitate labour). But, curiously, he concluded "that the spinal meningitic and myelitic affections may play a considerable part in the phenomena of spastic rigidity." His finale is a brief but optimistic reference to "therapeutic effects producing amelioration surprising to those who have not watched such cases."

JMS PEARCE

\section{References}

1 Little WJ. Course of Lectures on the deformities of the human frame. Lancet 1943;i:5-8; ibid 38-44; ibid 78-82 et seq.

2 Little WJ. On the nature and treatment of deformities of the human frame: being a course of lectures delivered at the Royal Orthopaedic Hospital in 1843: with numerous notes and additions to the present time. London: Longman, 1853.

3 Little WJ. On the influence of abnormal parturition, difficult labours, premature birth, and asphyxia neonatorum, on the mental and physical condition of the child especially in relation to deformities. Transactions of the Obstetrical Society of London, 1861;3:293-344. 